University of Nebraska - Lincoln

DigitalCommons@University of Nebraska - Lincoln

Faculty Publications from Nebraska Center for

Materials and Nanoscience, Nebraska Center Materials and Nanoscience

for (NCMN)

8-22-2007

\title{
Size effect in polymer nanofibers under tension
}

Xiangfa Wu

University of Nebraska - Lincoln, xwu3@unl.edu

Yuris A. Dzenis

University of Nebraska-Lincoln, ydzenis@unl.edu

Follow this and additional works at: https://digitalcommons.unl.edu/cmrafacpub

Part of the Nanoscience and Nanotechnology Commons

Wu, Xiangfa and Dzenis, Yuris A., "Size effect in polymer nanofibers under tension" (2007). Faculty Publications from Nebraska Center for Materials and Nanoscience. 32.

https://digitalcommons.unl.edu/cmrafacpub/32

This Article is brought to you for free and open access by the Materials and Nanoscience, Nebraska Center for (NCMN) at DigitalCommons@University of Nebraska - Lincoln. It has been accepted for inclusion in Faculty Publications from Nebraska Center for Materials and Nanoscience by an authorized administrator of DigitalCommons@University of Nebraska - Lincoln. 


\title{
Size effect in polymer nanofibers under tension
}

\author{
Xiang-Fa Wu ${ }^{\mathrm{a})}$ and Yuris A. Dzenis ${ }^{\mathrm{b})}$ \\ Department of Engineering Mechanics, Nebraska Center for Materials and Nanoscience, \\ University of Nebraska-Lincoln, Lincoln, Nebraska 68588-0526
}

(Received 25 May 2007; accepted 28 June 2007; published online 22 August 2007)

\begin{abstract}
This article studies the size effect on the elastic behavior of solid and hollow polymer nanofibers (e.g., electrospun nanofibers) subjected to uniaxial tension. A one-dimensional nonlinear elastic tension model is proposed that takes into account the coupling effect of fiber elastic deformation and surface tension. The fiber axial force-displacement and stress-strain relations are obtained in explicit forms. It is shown that, at nanoscale, fiber radius has appreciable effect on the elastic response of polymer nanofibers. With consideration of the fiber radial effect, it is shown that the actual contribution of surface energy of the solid polymer fibers to the axial tensile force is $\pi r_{0} \gamma$ rather than $2 \pi r_{0} \gamma$ (where $r_{0}$ is the fiber radius after deformation and $\gamma$ is the surface tension), as commonly used in literature. Compared to solid polymer fibers, the tensile behavior of hollow polymer nanofibers appears more complex with greater axial stiffening effect depending upon the combination effect of the fiber exterior and interior radii and the material properties. The results presented in this study can be utilized for data reduction of the nanoscale tension tests of polymer nanofibers and the analysis and design of nanofiber devices. (C) 2007 American Institute of Physics.
\end{abstract}

[DOI: $10.1063 / 1.2769266]$

\section{INTRODUCTION}

Due to their high porosity, strength, and surface area to volume ratio, fiber networks and fibrous materials have found extensive applications in thermal and sound insulators, gas and fluid filters, chemical carriers, tissue templates, and various paper products. In these materials and products, individual fibers are the key constituents and their mechanical properties play a crucial role in the global response of fibrous materials subjected to external loadings. As a matter of fact, the mechanical properties of fibrous materials can be remarkably enhanced through improving the properties of the individual fibers, the interface properties of fiber-fiber contacts, and the fiber lay-ups within the fibrous materials. Research has indicated that the mechanical properties of individual fibers (e.g., ultimate tensile strength, fatigue and damage tolerance, etc.) can be improved with decreasing fiber diameters under proper spinning conditions. Furthermore, recent ultrathin fibers produced by the electrospinning technique ${ }^{1-4}$ further extend the applications of classic fibrous materials. So far, continuous ultrathin fibers with diameters ranging from a few microns down to a few nanometers were fabricated efficiently by means of the electrospinning technique. With their ultrahigh surface area to volume ratio and tensile strength, continuous nanofibers and their derivatives are expected to form fiber networks with high porosity and tensile strength for the use of electromagnetic shielding, chemical catalyst carriers, tissue templates, artificial skins, nanofiber composites, etc. ${ }^{5-8}$

In applications of fibrous materials made of ultrathin fibers, the mechanical behavior of individual fibers is fundamental to their targeted functions. In recent years, this has

\footnotetext{
${ }^{a)}$ Electronic mail: xfwu@unlserve.unl.edu

${ }^{\mathrm{b})}$ Electronic mail: ydzenis@unl.edu
}

triggered considerable investigation in characterizing the mechanical properties of continuous nanofibers (e.g., Young's modulus ${ }^{9-15}$ ) and relevant mechanics modeling of fibers, fiber networks, and nanofiber composites. ${ }^{16-21}$ To mention a few, atomic force microscopy (AFM)-based uniaxial tension and three-point bending tests ${ }^{9-14}$ have been conducted recently to characterize the Young's modulus and ultimate tensile strength of electrospun polymer nanofibers with diameters ranging from $1 \mu \mathrm{m}$ down to hundreds of nanometers. In a typical uniaxial tension test, one end of the nanofiber is fastened on the surface of a silicon wafer by adhesive and the other is tethered to the AFM tip. The microscopic tensile force is exerted through the motion of the AFM tip. In the case of a three-point bending test, the nanofiber segment is clamped at two ends by adhesive on the surface of a silicon wafer with periodic gratings. The transverse bending force is induced by the AFM tip at the midspan of the nanofiber segment between two neighboring lines. Furthermore, the Young's modulus of nanofibers can also be determined dynamically through the natural frequencies of a pair of AFM microcantilevers connected by nanofiber segments. ${ }^{15}$ So far, experimental results available in the literature ${ }^{9-14}$ have indicated that the axial Young's modulus of electrospun polymer nanofibers varied with the change of the take-up speed and the nanofiber radius. For example, experiments found that the axial Young's modulus decreased with the increase of either the fiber radius or the take-up speed in electrospinning. This behavior may be attributed to the change of molecular chain orientation (crystallization) during fiber spinning and the increase of surface energy. Moreover, as one of the unique properties of nanomaterials, surface has appreciable effect on the stiffness of nanosized metallic and organic wires and films as was observed in experiments and also predicted by theoretical studies. ${ }^{22-28}$ For crystalline nanowires and ultrathin films surface stresses may either increase 


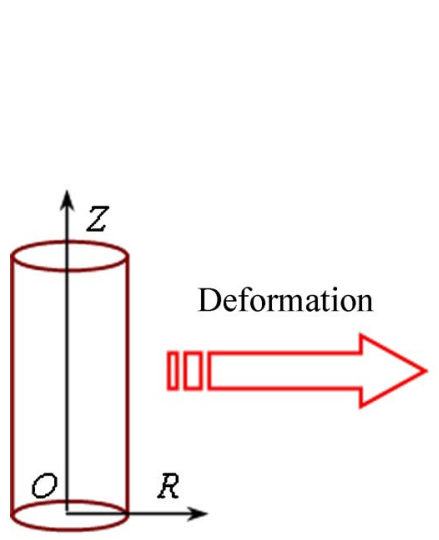

(a)

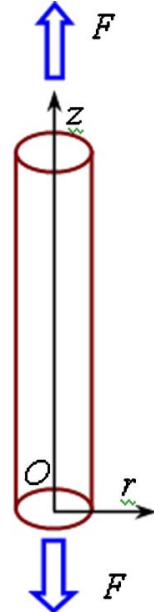

(b)

FIG. 1. The coordinate systems used for the analysis of solid cylindrical nanofibers under uniaxial stretching: (a) strain-free state and (b) state after deformation.

or decrease the corresponding moduli depending upon the specific material types and crystalline structures. For polymer materials, surface tension increased the moduli of ultrafine fibers and films as has been observed in experiments.

So far, besides a few experimental investigations on the axial mechanical behavior of electrospun polymer nanofibers, no work has been reported to disclose the mechanisms of the variation of their mechanical properties versus the fiber radius and other parameters. In this work we study the size effect on the elastic behavior of solid and hollow polymer nanofibers subjected to uniaxial stretching. Within the framework of nonlinear elasticity, a one-dimensional fiber tension model is proposed to take into account the coupling effect of fiber elastic deformation and surface tension. Explicit solutions for the fiber axial deformations are obtained. Numerical simulations are performed to show the effects of fiber radius and surface tension on the elastic behavior of polymer nanofibers subjected to axial stretching. Potential applications of the present model and results in nanoscale tension tests are further addressed.

\section{PROBLEM STATEMENT AND SOLUTIONS}

\section{A. Thin solid fibers under uniaxial stretching}

Consider a thin solid cylindrical polymer fiber subjected to uniaxial stretching as illustrated in Fig. 1. The polymer fiber is dealt with as hyperelastic isotropic material (incompressible), which is a good approach for rubble-like polymers. Within the framework of nonlinear elasticity, the motion of a material point inside the fiber can be described $\operatorname{as}^{29,30}$

$$
\begin{aligned}
& r=\lambda_{1} R \quad\left(0 \leq R \leq R_{0}\right), \\
& \theta=\Theta \quad(0 \leq \Theta \leq 2 \pi),
\end{aligned}
$$

$$
z=\lambda_{3} Z \quad(0 \leq Z \leq L)
$$

where $(r, \theta, z)$ are the spatial coordinates, $(R, \Theta, Z)$ are the material coordinates for the material point, and $\lambda_{1}$ and $\lambda_{3}$ are the principal stretches of the material element in the radial and axial directions, respectively. In the present case of incompressible material, $\lambda_{1}$ and $\lambda_{3}$ satisfy

$$
\lambda_{1}^{2} \lambda_{3}=1
$$

Therefore, the left Cauchy-Green tensor $\mathbf{B}$ and its inverse can be expressed as

$$
\begin{aligned}
& \mathbf{B}=\mathbf{F F}^{T}=\operatorname{diag}\left[\lambda_{1}^{2}, \lambda_{1}^{2}, \lambda_{3}^{2}\right], \\
& \mathbf{B}^{-1}=\operatorname{diag}\left[\lambda_{1}^{-2}, \lambda_{1}^{-2}, \lambda_{3}^{-2}\right],
\end{aligned}
$$

where $\mathbf{F}$ is the deformation gradient

$$
\mathbf{F}=\left[\begin{array}{ccc}
\partial r / \partial R & 1 / R \partial r / \partial \Theta & \partial r / \partial Z \\
r \partial \theta / \partial R & r / R \partial \theta / \partial \Theta & r \partial \theta / \partial Z \\
\partial z / \partial R & 1 / R \partial z / \partial \Theta & \partial z / \partial Z
\end{array}\right] .
$$

The scalar invariants of $\mathbf{B}$ are

$$
\begin{aligned}
& I_{1}=2 \lambda_{1}^{2}+\lambda_{3}^{2}=\frac{2}{\lambda_{3}}+\lambda_{3}^{2}, \\
& I_{2}=2 \lambda_{3}+\frac{1}{\lambda_{3}^{2}}, \\
& I_{3}=1 .
\end{aligned}
$$

The constitutive law of the hyperelastic isotropic material can be expressed in terms of Cauchy stresses versus $\mathbf{B}$,

$$
\mathbf{T}=-p \mathbf{I}+c_{1} \mathbf{B}+c_{2} \mathbf{B}^{-1},
$$

where $p$ is the hydrostatic pressure and $c_{1}$ and $c_{2}$ are two independent material constants. In terms of components, it reads

$$
\begin{aligned}
& T_{r r}=T_{\theta \theta}=-p+c_{1} \lambda_{1}^{2}+\frac{c_{2}}{\lambda_{1}^{2}}=-p+\frac{c_{1}}{\lambda_{3}}+c_{2} \lambda_{3}, \\
& T_{z z}=-p+c_{1} \lambda_{3}^{2}+c_{2} \lambda_{1}^{4}=-p+c_{1} \lambda_{3}^{2}+\frac{c_{2}}{\lambda_{3}^{2}},
\end{aligned}
$$

$T_{r \theta}=T_{r z}=T_{\theta z}=0$.

In the spatial coordinate system, equations of equilibrium lead to

$$
\begin{aligned}
& \frac{\partial T_{r r}}{\partial r}+\frac{T_{r r}-T_{\theta \theta}}{r}=0, \\
& \frac{\partial T_{\theta \theta}}{\partial \theta}=0,
\end{aligned}
$$

$$
\frac{\partial T_{z z}}{\partial z}=0
$$


Equations (7) and (10) imply that $T_{r r}$ and $T_{\theta \theta}$ are independent of fiber radius. Furthermore, since the scalar invariants $I_{1}$ and $I_{2}$ in Eq. (5) are only functions of $r$, Eqs. (11) and (12) result in

$$
\frac{\partial p}{\partial \theta}=\frac{\partial p}{\partial z}=0,
$$

i.e., the hydrostatic pressure only varies with respect to $r$,

$$
p=p(r) \text {. }
$$

The total axial tensile force $P$ can be evaluated as

$$
P=\int_{0}^{r_{0}} T_{z z} 2 \pi r d r+2 \pi r_{0} \gamma,
$$

where $r_{0}$ is the current fiber radius after stretching, $\gamma$ is the surface tension, and $T_{z z}$ can be determined by eliminating $p$ in Eqs. (7) and (8) and using Eq. (10)

$$
T_{z z}=\frac{1}{2 r} \frac{d\left(r^{2} T_{r r}\right)}{d r}+\left(\lambda_{3}^{2}-\frac{1}{\lambda_{3}}\right)\left(c_{1}-\frac{c_{2}}{\lambda_{3}}\right) .
$$

Substitution of Eq. (16) into Eq. (15) yields

$$
P=\left.\pi r^{2} T_{r r}\right|_{0} ^{r_{0}}+\pi r_{0}^{2}\left(\lambda_{3}^{2}-\frac{1}{\lambda_{3}}\right)\left(c_{1}-\frac{c_{2}}{\lambda_{3}}\right)+2 \pi r_{0} \gamma \text {. }
$$

With the aid of the boundary traction condition with surface tension

$$
T_{r r}\left(r_{0}\right)=-\gamma / r,
$$

relation (17) can be rewritten as

$$
P=\pi r_{0}^{2}\left(\lambda_{3}^{2}-\frac{1}{\lambda_{3}}\right)\left(c_{1}-\frac{c_{2}}{\lambda_{3}}\right)+\pi r_{0} \gamma
$$

and the corresponding true axial tensile stress is

$$
\sigma_{z z}=\frac{P}{\pi r_{0}^{2}}=\left(\lambda_{3}^{2}-\frac{1}{\lambda_{3}}\right)\left(c_{1}-\frac{c_{2}}{\lambda_{3}}\right)+\frac{\gamma}{r_{0}} .
$$

Since $r_{0}=\lambda_{1} R_{0}=R_{0} / \sqrt{\lambda_{3}}$, relations (19) and (20) can be recast into the original material configuration as

$$
\begin{aligned}
& P=\pi R_{0}^{2}\left(\lambda_{3}-\frac{1}{\lambda_{3}^{2}}\right)\left(c_{1}-\frac{c_{2}}{\lambda_{3}}\right)+\frac{\pi R_{0} \gamma}{\sqrt{\lambda_{3}}}, \\
& \sigma_{z z}=\frac{P}{\pi R_{0}^{2}}=\left(\lambda_{3}-\frac{1}{\lambda_{3}^{2}}\right)\left(c_{1}-\frac{c_{2}}{\lambda_{3}}\right)+\frac{\gamma}{R_{0} \sqrt{\lambda_{3}}} .
\end{aligned}
$$

It can be observed from Eq. (19) that when taking into account the radial effect, the actual contribution of fiber surface energy to the axial tensile force is $\pi r_{0} \gamma$ rather than $2 \pi r_{0} \gamma$ as commonly used in literature.

\section{B. Thin hollow fibers under uniaxial stretching}

Now let us consider the finite deformation of a thin hollow cylindrical fiber subjected to uniaxial stretching as shown in Fig. 2. The initial interior and exterior radii of the fiber are denoted as $R_{A}$ and $R_{B}$, respectively. The fiber material is considered as hyperelastic isotropic similar to Sec.

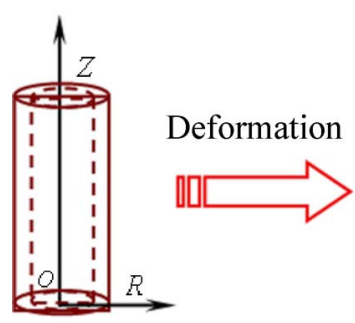

(a)

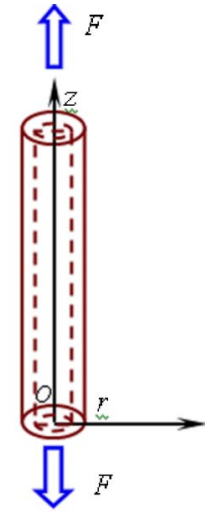

(b)
FIG. 2. The coordinate systems used for the analysis of hollow nanofibers under uniaxial stretching: (a) strain-free state and (b) state after deformation.

II A. In this case, by introducing the coordinate systems earlier, the motion of a material point can be described as ${ }^{29}$

$$
\begin{aligned}
& r=R f(R) \quad\left(R_{A} \leq R \leq R_{B}\right), \\
& \theta=\Theta \quad(0 \leq \Theta \leq 2 \pi), \\
& z=\lambda_{3} Z \quad(0 \leq Z \leq L),
\end{aligned}
$$

where $\lambda_{3}$ is the axial stretch which is a constant at fixed axial loading. Therefore, the left Cauchy-Green tensor $\mathbf{B}$ and its inverse in this case can be expressed as

$$
\begin{aligned}
& \mathbf{B}=\mathbf{F} \mathbf{F}^{T}=\operatorname{diag}\left\{\left[R f^{\prime}(R)+f(R)\right]^{2},[f(R)]^{2}, \lambda_{3}^{2}\right\}, \\
& \mathbf{B}^{-1}=\operatorname{diag}\left\{\left[R f^{\prime}(R)+f(R)\right]^{-2},[f(R)]^{-2}, \lambda_{3}^{-2}\right\} .
\end{aligned}
$$

Within the coordinate system $(R, \Theta, Z)$, the principal stretches along the radial and axial directions are

$$
\begin{aligned}
& \lambda_{1}=R f^{\prime}(R)+f(R), \\
& \lambda_{2}=f(R), \\
& \lambda_{3}=\text { constant } .
\end{aligned}
$$

Material incompressibility of the polymer fiber leads to

$$
f(R)\left[R f^{\prime}(R)+f(R)\right]=1 / \lambda_{3} .
$$

The solution to the earlier equation yields

$$
f(R)=\sqrt{1 / \lambda_{3}+A / R^{2}},
$$

in which $A$ is an unknown constant to be determined. Substitution of Eqs. (24) and (25) into the constitutive relation (6) gives the stress components

$$
T_{r r}=-p+c_{1}\left[R f^{\prime}(R)+f(R)\right]^{2}+c_{2}\left[R f^{\prime}(R)+f(R)\right]^{-2},
$$

$$
\begin{aligned}
& T_{\theta \theta}=-p+c_{1}[f(R)]^{2}+c_{2}[f(R)]^{-2}, \\
& T_{z z}=-p+c_{1} \lambda_{3}^{2}+c_{2} \lambda_{3}^{-2}, \\
& T_{r \theta}=T_{r z}=T_{\theta z}=0 .
\end{aligned}
$$


The equations of equilibrium are Eqs. (10)-(12). Again, Eqs. (11) and (12) guarantee that the hydrostatic stress $p$ is only a function with respect to $r$. The unknown constant $A$ in Eq. (28) can be determined by integrating Eq. (10) with respect to $r$,

$$
\left.T_{r r}\right|_{r_{A}} ^{r_{B}}+\int_{r_{A}}^{r_{B}}\left(T_{r r}-T_{\theta \theta}\right) / r d r=0 .
$$

With the radial traction boundary conditions: $T_{r r}\left(r_{A}\right)$ $=\gamma / r_{A}$ and $T_{r r}\left(r_{B}\right)=-\gamma / r_{B}$, substituting Eqs. (29) and (30) into Eq. (33) leads to

$$
\begin{aligned}
\gamma\left[\frac{1}{R_{A} f\left(R_{A}\right)}+\frac{1}{R_{B} f\left(R_{B}\right)}\right]= & \int_{R_{A}}^{R_{B}}\left\{c_{1}\left[R f^{\prime}(R)+f(R)\right]^{3}\right. \\
& \left.+\frac{c_{2}}{R f^{\prime}(R)+f(R)}\right\} \frac{1}{R f(R)} d R \\
& -\int_{R_{A}}^{R_{B}}\left\{c_{1}[f(R)]^{2}\right. \\
& \left.+\frac{c_{2}}{[f(R)]^{2}}\right\} \frac{R f^{\prime}(R)+f(R)}{R f(R)} d R .
\end{aligned}
$$

Thus, the unknown constant $A$ can be determined by solving the earlier nonlinear equation numerically as demonstrated in Sec. III.

Furthermore, similar to Eq. (15), the total axial tensile force $P$ can be determined as

$$
P=\int_{r_{A}}^{r_{B}} T_{z z} 2 \pi r d r+2 \pi\left(r_{A}+r_{B}\right) \gamma .
$$

In order to evaluate Eq. (35), one needs first to eliminate $p$ from $T_{z z}$ in Eq. (31). This can be fulfilled as follows. ${ }^{30}$

Rewriting Eqs. (29)-(31) as

$$
\begin{aligned}
& T_{r r}=-p+\tau_{r r}, \\
& T_{\theta \theta}=-p+\tau_{\theta \theta}, \\
& T_{z z}=-p+\tau_{z z},
\end{aligned}
$$

gives

$$
2 T_{z z}=T_{r r}+T_{\theta \theta}+2 \tau_{z z}-\tau_{r r}-\tau_{\theta \theta} .
$$

Substitutions of $T_{\theta \theta}$ from Eq. (10) into Eq. (37) leads to

$$
2 T_{z z}=\frac{1}{r} \frac{d r^{2} T_{r r}}{d r}+2 \tau_{z z}-\tau_{r r}-\tau_{\theta \theta}
$$

With the help of Eqs. (37) and (38), the axial tensile force Eq. (35) can be recast into

$$
\begin{aligned}
P= & \left.\pi r^{2} T_{r r}\right|_{r_{A}} ^{r_{B}}+\pi \int_{r_{A}}^{r_{B}}\left(2 \tau_{z z}-\tau_{r r}-\tau_{\theta \theta}\right) r d r+2 \pi\left(r_{A}\right. \\
& \left.+r_{B}\right) \gamma .
\end{aligned}
$$

With the radial traction boundary conditions: $T_{r r}\left(r_{A}\right)$ $=\gamma / r_{A}$ and $T_{r r}\left(r_{B}\right)=-\gamma / r_{B}$, relation (39) can be rewritten as

$$
P=\pi \int_{r_{A}}^{r_{B}}\left(2 \tau_{z z}-\tau_{r r}-\tau_{\theta \theta}\right) r d r+\pi\left(r_{A}+r_{B}\right) \gamma
$$

and the true axial stress is

$$
\sigma_{z z}=\frac{\int_{r_{A}}^{r_{B}}\left(2 \tau_{z z}-\tau_{r r}-\tau_{\theta \theta}\right) r d r}{r_{B}^{2}-r_{A}^{2}}+\frac{r_{A}+r_{B}}{r_{B}^{2}-r_{A}^{2}} \gamma .
$$

In the material configuration, relations (40) and (41) can be rewritten as

$$
\begin{aligned}
P= & \pi \int_{R_{A}}^{R_{B}}\left(2 \tau_{z z}-\tau_{r r}-\tau_{\theta \theta}\right) R f(R)\left[R f^{\prime}(R)+f(R)\right] d R \\
& +\pi \gamma\left[R_{A} f\left(R_{A}\right)+R_{B} f\left(R_{B}\right)\right], \\
\sigma_{z z}= & \frac{\int_{R_{A}}^{R_{B}}\left(2 \tau_{z z}-\tau_{r r}-\tau_{\theta \theta}\right) R f(R)\left[R f^{\prime}(R)+f(R)\right] d R}{\left[R_{B} f\left(R_{B}\right)\right]^{2}-\left[R_{A} f\left(R_{A}\right)\right]^{2}} \\
& +\gamma \frac{R_{A} f\left(R_{A}\right)+R_{B} f\left(R_{B}\right)}{\left[R_{B} f\left(R_{B}\right)\right]^{2}-\left[R_{A} f\left(R_{A}\right)\right]^{2}} .
\end{aligned}
$$

From Eqs. (29)-(31) and (37), we have

$$
\begin{aligned}
2 \tau_{z z}-\tau_{r r}-\tau_{\theta \theta}= & c_{1}\left\{2 \lambda_{3}^{2}-\left[R f^{\prime}(R)+f(R)\right]^{2}-[f(R)]^{2}\right\} \\
& +c_{2}\left\{2 \lambda_{3}^{-2}-\left[R f^{\prime}(R)+f(R)\right]^{-2}\right. \\
& \left.-[f(R)]^{-2}\right\} .
\end{aligned}
$$

Therefore, once the fiber material constants $c_{1}$ and $c_{2}$, surface tension $\gamma$, initial interior and exterior radii $R_{A}$ and $R_{B}$, and axial stretch $\lambda_{3}$ are given, the unknown constant $A$ can be determined from Eq. (34). With the constant $A$, the forcestretch and stress-strain relations can be determined conveniently by numerical integration of Eqs. (40) and (41) in the material configuration.

\section{NUMERICAL EXAMPLES AND DISCUSSIONS}

Let us consider numerical examples to examine the size effect in the elastic behavior of the solid and hollow polymer nanofibers with varying radius. The elastic constants of the fiber material $c_{1}$ and $c_{2}$ are selected to be close to those of vulcanized rubber compounds ${ }^{31} c_{1}=0.1-0.31 \mathrm{MPa}, c_{2}=$ $-0.1 \mathrm{MPa}$, and the surface tension $\gamma$ is $\gamma=0.05-0.1 \mathrm{~N} / \mathrm{m}$.

Figure 3 shows the variation of the true axial stress versus the axial stretch $\lambda_{3}$ of the solid fibers with varying fiber radius based on relation (20). In this case, the material properties used in the calculation are $c_{1}=0.1 \mathrm{MPa}, c_{2}=$ $-0.1 \mathrm{MPa}$, and $\gamma=0.05 \mathrm{~N} / \mathrm{m}$. At small stretch, $\lambda_{3} \approx 1+\varepsilon_{33}$, where $\varepsilon_{33}$ is the axial linear tensile strain. It can be observed that surface tension has significant effect on the initial axial stress of fibers with decreasing fiber radius. For fiber radius greater than $500 \mathrm{~nm}$, the size effect is almost unappreciable. It can also be found that the axial stiffness of nanofibers increases appreciably with the decrease of the fiber radius. Due to the existence of surface tension, finite axial tensile traction is required at initial stretching. 


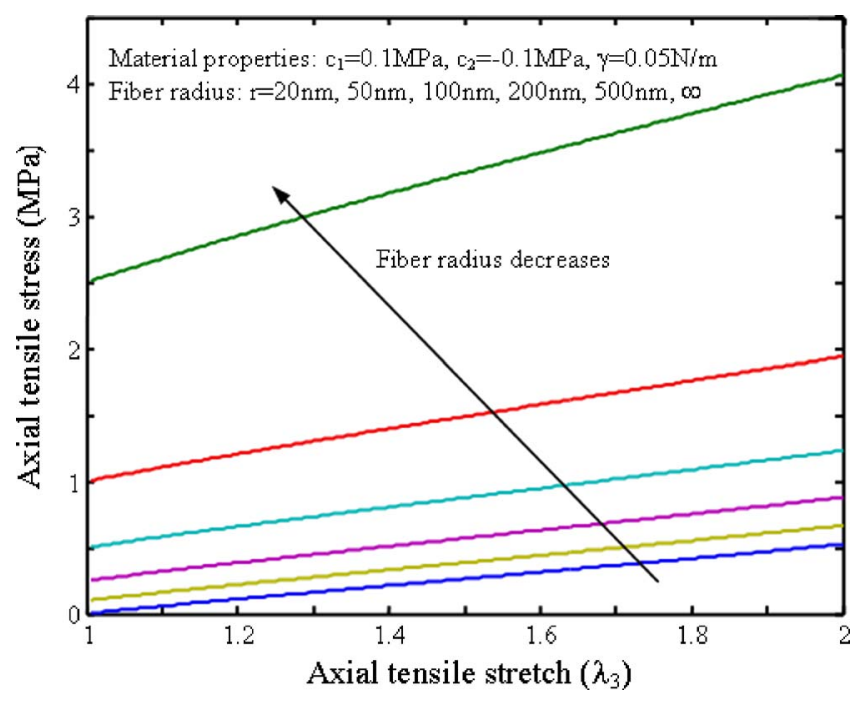

FIG. 3. Variation of the true axial tensile stress of solid nanofibers vs the axial tensile stretch at varying fiber radius.

Figure 4 shows the variation of the true axial stress versus the axial stretch $\lambda_{3}$ of the hollow fibers with varying fiber radius according to relation (43). In this case, the ratio of the interior radius to the exterior one is fixed as 0.5 in the entire numerical process. For the purpose of comparison, the material properties are chosen as those of solid fibers shown in Fig. 3. The numerical process consists of solving the nonlinear Eq. (34) to extract the constant $A$ and the numerical integration of Eq. (43) to determine the true axial tensile stress at given stretch $\lambda_{3}$ and original exterior radius $R_{B}$. Similar to Fig. 3, results plotted in Fig. 4 show that surface tension has significant effect on the initial axial tensile stress of the nanofibers with decreasing fiber radius. Compared to the results for solid fibers (see Fig. 3), at the same fiber exterior radius hollow fibers appear to have higher axial stiffness due to the extra surface tension and internal constraints induced by the hollow structure. Furthermore, with the increase of axial stretch, the axial stiffness of hollow fibers grows faster

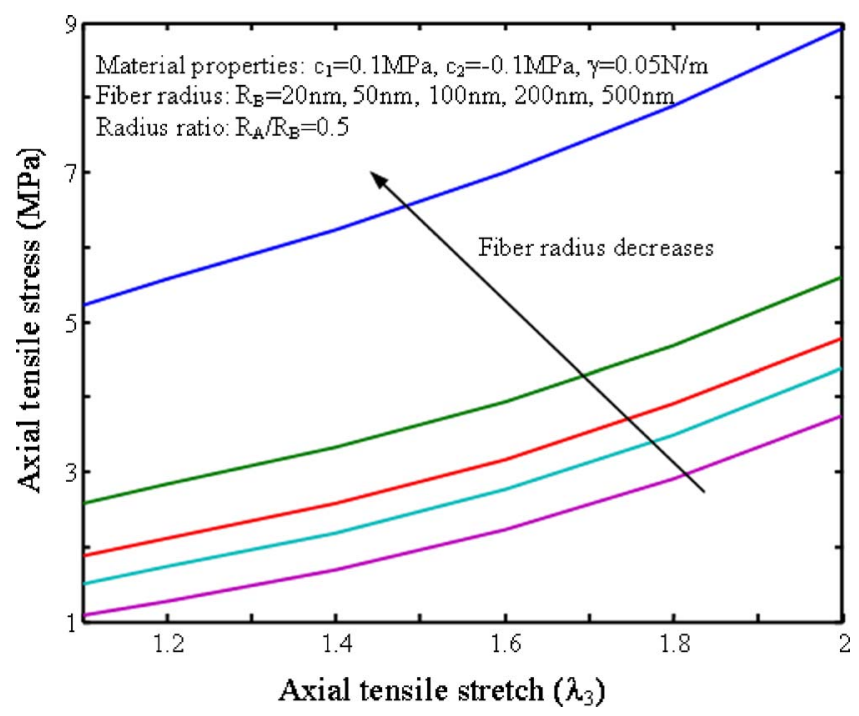

FIG. 4. Variation of the true axial tensile stress of hollow nanofibers vs the axial tensile stretch at varying fiber radius. than that of solid fibers with the same radii. Therefore, hollow fibers exhibit more axial stiffening effect at the nanoscale.

\section{CONCLUDING REMARKS}

One-dimensional nonlinear elastic model involving finite deformations and surface tension has been developed in this study. The model has considered the coupling effect of surface tension and fiber radius on the tensile response of solid and hollow polymer nanofibers. The fiber material was assumed to be rubber-like hyperelastic isotropic material that is close to the typical as-spun polymer nanofibers in electrospinning. The present study shows that for solid nanofibers, the effect of surface tension is decoupled, i.e., it is independent of the material properties as indicated in Eq. (20). However, for hollow nanofibers, the effect of surface tension is coupled with the nanofiber geometry and the material properties through the constant $A$ in Eq. (34). Therefore, the finite stretching of hollow nanofibers exhibits more complex elastic behavior. Due to involvement of the surface tension, finite axial tensile force is required for the initial axial stretching of nanofibers.

The present study shows that at nanoscale fiber radius has appreciable effect on the elastic response of polymer nanofibers. With consideration of the fiber radial effect, the actual contribution of surface energy of the solid fibers to the axial tensile force is $\pi r_{0} \gamma$ rather than $2 \pi r_{0} \gamma$ as used commonly in literature. The tensile stretching of hollow nanofibers appears more complex and depends on the fiber geometry and material properties as indicated in relation (43). At nanoscale, hollow nanofibers show greater axial stiffening effect with increasing axial stretch.

Nanofibers are being considered for nanofiber devices and sensors. Due to their ultrathin structure, nanofibers are very flexible and can exhibit large deformations. Thus, the discovered size effect on their deformation should be taken into account for precise prediction of their properties and mechanical response. Finally, the results of the present study can be employed for data reduction of the nanoscale tension tests of polymer nanofibers and for analysis and design of nanofiber devices.

\section{ACKNOWLEDGMENTS}

Partial support of this work by the NSF, U.S. AFOSR, and U.S. ARO/ARL is gratefully acknowledged.

${ }^{1}$ J. Doshi and D. H. Reneker, J. Electrost. 35, 151 (1995).

${ }^{2}$ D. H. Reneker and I. Chun, Nanotechnology 7, 216 (1996).

${ }^{3}$ Y. Dzenis, Science 304, 1917 (2004).

${ }^{4}$ D. Li and Y. N. Xia, Adv. Mater. 16, 1151 (2004).

${ }^{5}$ J. S. Kim and D. H. Reneker, Polym. Compos. 20, 124 (1999).

${ }^{6}$ Z. M. Huang, Y. Z. Zhang, M. Kotaki, and S. Ramakrishna, Compos. Sci. Technol. 63, 2223 (2003).

${ }^{7}$ S. Y. Chew, Y. Wen, Y. Dzenis, and K. W. Leong, Curr. Pharm. Des. 12, 4751 (2006).

${ }^{8}$ W. E. Teo and S. Ramakrishna, Nanotechnology 17, R89 (2006)

${ }^{9}$ E. P. S. Tan and C. T. Lim, Rev. Sci. Instrum. 75, 2581 (2004).

${ }^{10}$ E. P. S. Tan, C. N. Goh, C. H. Sow, and C. T. Lim, Appl. Phys. Lett. 86, 073115 (2005).

${ }^{11}$ E. P. S. Tan, S. Y. Ng, and C. T. Lim, Biomaterials 26, 1453 (2005)

${ }^{12}$ R. Inai, M. Kotaki, and S. Ramakrishna, Nanotechnology 16, 208 (2005). 
${ }^{13}$ E. P. S. Tan and C. T. Lim, Compos. Sci. Technol. 66, 1102 (2006).

${ }^{14}$ E. Zussman, M. Burman, A. L. Yarin, R. Khalfin, and Y. Cohen, J. Polym. Sci., Part B: Polym. Phys. 44, 1482 (2006).

${ }^{15}$ P. A. Yuya, Y. K. Wen, J. A. Turner, and Y. A. Dzenis, Appl. Phys. Lett. 90, 111909 (2007).

${ }^{16}$ X. F. Wu and Y. A. Dzenis, J. Appl. Phys. 98, 093501 (2005).

${ }^{17}$ X. F. Wu and Y. A. Dzenis, J. Appl. Phys. 100, 124318 (2006).

${ }^{18}$ A. P. Chatterjee, J. Appl. Phys. 100, 054302 (2006).

${ }^{19}$ A. P. Chatterjee and D. A. Prokhorova, J. Appl. Phys. 101, 104301 (2007).

${ }^{20}$ X. F. Wu and Y. A. Dzenis, Nanotechnology 18, 285702 (2007).

${ }^{21}$ X. F. Wu and Y. A. Dzenis, J. Phys. D 40, 4276 (2007).

${ }^{22}$ R. C. Cammarata and K. Sieradzki, Phys. Rev. Lett. 62, 2005 (1989).
${ }^{23}$ P. Gumbsch and M. S. Daw, Phys. Rev. B 44, 3934 (1991).

${ }^{24}$ F. H. Streitz, R. C. Cammarata, and K. Sieradzki, Phys. Rev. B 49, 10699 (1994).

${ }^{25}$ R. C. Cammarata, Prog. Surf. Sci. 46, 1 (1994)

${ }^{26}$ R. E. Miller and V. B. Shenoy, Nanotechnology 11, 139 (2000).

${ }^{27}$ A. Sharma and G. Reiter, J. Colloid Interface Sci. 178, 383 (1996).

${ }^{28}$ X. F. Wu and Y. A. Dzenis, J. Phys. D 38, 2848 (2005).

${ }^{29}$ R. W. Ogden, Non-Linear Elastic Deformations (Dover, New York, 1997).

${ }^{30}$ W. M. Lai, D. Rubin, and E. Krempl, Introduction to Continuum Mechanics (Butterworth-Hernemann, Woburn, 1993).

${ }^{31}$ L. T. G. Trelorar, The Physics of Rubber Elasticity (Clarendon, Oxford, 1975). 